\title{
Influencia de hongos endomicorrízicos en el crecimiento de (Tabebuia donnell-smithii Rose)
}

\section{Influence of endomycorrhizal fungi on the growth of (Tabebuia donnell-smithii Rose)}

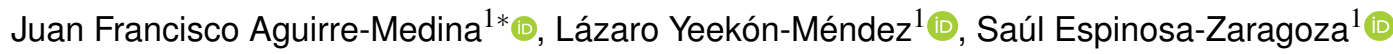 \\ ${ }^{1}$ Universidad Autónoma de Chiapas, Facultad de Ciencias Agrícolas Campus IV, Entronque carretera costera y Huehuetan Pueblo, Km 1, \\ CP. 30660. Municipio de Huehuetán, Chiapas, México. \\ *Autor de correspondencia: juanf56@prodigy.net.mx
}

Artículo científico recibido: 01 de julio de 2017 aceptado: 14 de julio de 2018

RESUMEN. Con el objetivo de identificar la influencia de hongos endomicorrízicos en el crecimiento de Tabebuia donnellsmithii Rose se estableció un experimento en invernadero. Se utilizaron colectas de hongos endomicorrízicos de Tenampulco Puebla y San Rafael Veracruz, y el hongo de referencia Rhizophagus intraradices, las cuales se evaluaron con suelo fluvisoleútrico en combinación con arena $(1: 1 \mathrm{v} / \mathrm{v})$, turba $(0.80: 0.20 \mathrm{v} / \mathrm{v})$ y estiércol bovino $(0.80: 0.20 \mathrm{v} / \mathrm{v})$, con los sustratos se llenaron bolsas de plástico de $5 \mathrm{~kg}$. Se evaluaron nueve tratamientos con la combinación de los sustratos y hongos, bajo un diseño completamente al azar con cuatro repeticiones. Se determinaron las variables morfológicas, fisiológicas, colonización radical y el contenido de fósforo en el tejido vegetal a los 112 días después de la siembra (dds). Los resultados indican incremento de $121 \%$ de la biomasa total en el sustrato adicionado con suelo más estiércol bovino. Entre microorganismos $R$. intraradices aumentó en 10.8 y $6.0 \%$ la biomasa en relación con las colectas caracoles y San Rafael, respectivamente. La biofertilización con $R$. intraradices promovió el crecimiento del tallo y el área foliar, y con el aislamiento caracoles, la altura y el área foliar. El mayor contenido de fósforo en comparación con las plantas con aislamientos endomicorrízicos nativos se presentó con $R$. intraradices.

Palabras clave: Micorriza arbuscular, biofertilización, primavera

ABSTRACT. A greenhouse experiment was conducted to identify the influence of endomycorrhizal fungi on the growth of Tabebuia donnell-smithii Rose. We used collections of endomycorrhizal fungi from Tenampulco, Puebla and San Rafael, Veracruz and the reference fungus Rhizophagus intraradices, which were evaluated with eutric fluvisol soil in combination with sand $(1: 1 \mathrm{v} / \mathrm{v})$, peat moss $(0.80: 0.20 \mathrm{v} / \mathrm{v})$ and bovine manure $(0.80: 0.20 \mathrm{v} / \mathrm{v}) ; 5-\mathrm{kg}$ plastic bags were filled with the substrates. Nine treatments were evaluated with the combination of substrates and fungi, under a completely randomized design with four replicates. Morphological, physiological, root colonization and phosphorus content variables were determined in plant tissue 112 days after sowing (das). Results indicate a 121\% increase in total biomass in the substrate with soil plus bovine manure added. Among microorganisms, $R$. intraradices increased biomass by 10.8 and $6.0 \%$ in relation to the snail and San Rafael collections, respectively. Biofertilization with $R$. intraradices promoted stem and leaf area growth, and with the snail isolates, height and leaf area. The highest phosphorus content compared to plants with native endomycorrhizal isolates occurred with $R$. intraradices.

Key words: Arbuscular mycorrhiza, biofertilization, spring.

\section{INTRODUCCIÓN}

Los hongos endomicorrízicos interactúan simbióticamente con más del $80 \%$ de las plantas terrestres (Gianinazzi et al. 2010). Es la simbiosis más común en la tierra y de las más antiguas (Wang y Qui 2006). Forma parte importante en el desarrollo, mantenimiento y estabilidad de los ecosistemas y representa un importante mecanismo para el desarrollo vegetal y reproductivo (Read 1998).

Las especies maderables de interés comercial como Tabebuia donnell-smithii Rose son propagadas de forma masiva en vivero mediante procedimientos rápidos con diferentes sustratos (Aguirre-Medina et 
al. 2014). Pero estos procedimientos no consideran el fortalecimiento del desarrollo radical de la planta hospedera con la biofertilización (Aguirre-Medina et al. 2012). Al aplicar los hongos endomicorrízicos se coloniza la raíz e inicia el intercambio de carbono de la planta hospedera al hongo, y del suelo se facilita el transporte de fósforo, agua y otros nutrimentos a la planta (Smith et al. 2015).

Los biofertilizantes con hongos endomicorrízicos favorecen el desarrollo vegetal y reproductivo de cultivos anuales y perennes, por ejemplo en los cultivos biofertilizados, se ha identificado mayor concentración de fósforo y nitrógeno en la planta (Aguirre-Medina 2006). Además de la activación de mecanismos de defensa contra patógenos (JaizmeVega y Rodríguez-Romero 2008), mejora la tolerancia de la planta en condiciones adversas (Doubková et al. 2013), y en condiciones de campo incrementa la supervivencia (Andrade et al. 2009). La asociación micorrízica es generalmente inespecífica, y el comportamiento de las poblaciones se modula por factores ambientales con evidencia de que tienen especificidad ecológica o sinergismo (Serralde y Ramírez 2004). Por lo anterior, el objetivo fue identificar la influencia de dos aislamientos de Rhizophagus intraradices, en los componentes morfológicos y fisiológicos de Tabebuia donnell-smithii Rose en diversos sustratos.

\section{MATERIALES Y MÉTODOS}

La investigación se realizó en la primavera de 2015 en el Campo Experimental de la Facultad de Ciencias Agrícolas Campus-IV, Huehuetán Chiapas. El vivero se localiza a los $15^{\circ} 00^{\prime} \mathrm{LN}$ y $94^{\circ} 30^{\prime} \mathrm{LO}$ a $44 \mathrm{~m}$ de altitud. La precipitación promedio es de 2 $326 \mathrm{~mm}$ anuales con temperatura máxima de 38, y mínima de $15^{\circ} \mathrm{C}$.

La semilla de Tabebuia donnell-smithii Rose se cosechó de árboles dispersos en el municipio de Tuxtla Chico, Chiapas, en la localidad Rosario Izapa (14 $30^{\circ}$ ' LN y $92^{\circ} 00^{\prime}$ LO). Los aislamientos de los hongos endomicorrízicos se obtuvieron en dos sitios, uno en caracoles (Tenampulco, Puebla, $20^{\circ} 08^{\prime} 30^{\prime \prime}$ LN y $97^{\circ} 30^{\prime} 00^{\prime \prime}$ LO a $350 \mathrm{msnm}$, con $2100 \mathrm{~mm}$ de precipitación y suelos Regosoles) y el otro en San Rafael (San Rafael, Veracruz, $20^{\circ} 08^{\prime} 20^{\prime \prime} \mathrm{LN}$ y $97^{\circ} 02^{\prime}$ 57" LO a $20 \mathrm{msnm}$, con $1600 \mathrm{~mm}$ de precipitación y suelos Cambisoles), ambos con cultivo de Vanilla planifolia Andrews en un ecosistema poco perturbado de selva alta perennifolia. En las muestras de raíces colectadas se verificó la presencia de hongos endomicorrízicos del género Glomus spp mediante tinción y clareo (Phillips y Hayman 1970).

Las esporas se separaron en cada muestra de suelo por grupos, mediante tamizado húmedo y decantación (Gerdermann y Nicholson 1963) de acuerdo al color, forma y tamaño, con un estéreo microscopio, y se propagaron por 90 días en sustrato de arena de río esterilizada (15 PSI a $250{ }^{\circ} \mathrm{C}$ por 20 min) con Sorghum bicolor (L.) Moench como planta hospedera en contenedores de un litro. A la cosecha se tuvieron al menos 100 esporas por gramo de suelo con el $93 \%$ de colonización radical. El hongo endomicorrízico de referencia $R$. intraradices (Schenck et Sm.) Walker et Schuessler contenía 40 esporas por gramo de suelo y $95 \%$ de colonización radical en la planta hospedera Brachiaria brizantha (Hochst. Ex. A. Rich.).

El análisis físico-químico de los sustratos (Tabla 1) se determinó por los siguientes métodos: Textura (Hidrómetro de Bouyucos), Densidad Aparente (DA, Probeta), Densidad real (DR, Picnómetro), \% Poros (Cálculo matemático), pH (Potenciómetro), Conductividad eléctrica (CE, Conductómetro), Nitrógeno (N, Micro-Kjeldahl), Fósforo ( $P$, Colorimetría), Potasio (K, Espectrofotometría de absorción atómica), Materia orgánica (MO, WalkleyBlack). El suelo utilizado se obtuvo a profundidad de $0-30 \mathrm{~cm}$ en terrenos del mismo Campo experimental, el cual es del grupo fluvisol eútrico (INEGI 2005). También se usó arena de río lavada, turba (peat moss) y estiércol bovino molido y esterilizado (15 PSI a $250{ }^{\circ} \mathrm{C}$ por $20 \mathrm{~min}$ ).

Los tratamientos fueron la combinación de los factores, sustrato y hongo endomicorrízico. Los sustratos fueron: 1) suelo más arena de río lavada en proporción ( $\mathrm{v} / \mathrm{v}) 1: 1,2)$ suelo más $20 \%$ de turba (producto comercial), 3) suelo más $20 \%$ de estiércol bovino; mientras que los microorganismos fueron: 1) 
Tabla 1. Características físico-químicas de los sustratos utilizados en el experimento.

\begin{tabular}{cccccccccc}
\hline Sustrato & $\begin{array}{c}\mathrm{DA} \\
\mathrm{g} / \mathrm{cm}^{3}\end{array}$ & $\begin{array}{c}\mathrm{DR} \\
\mathrm{g} / \mathrm{ml}\end{array}$ & $\begin{array}{c}\text { Poros } \\
\%\end{array}$ & $\mathrm{pH}$ & $\begin{array}{c}\mathrm{CE} \\
\mathrm{ds} \mathrm{m} \mathrm{m}^{-1}\end{array}$ & $\begin{array}{c}\mathrm{N} \\
(\%)\end{array}$ & $\begin{array}{c}\mathrm{P} \\
(\mathrm{ppm})\end{array}$ & $\begin{array}{c}\mathrm{K} \\
(\mathrm{ppm})\end{array}$ & $\begin{array}{c}\mathrm{MO} \\
\%\end{array}$ \\
\hline Suelo:arena & 1.6 & 2.08 & 23.07 & 6.5 & 0.05 & 0.10 & 33 & 11 & 1.9 \\
Suelo:turba & 1.1 & 1.85 & 38.30 & 6.2 & 0.10 & 0.43 & 81 & 24 & 8.5 \\
Suelo:estiércol & 1.2 & 1.88 & 33.50 & 6.8 & 0.86 & 0.43 & 179 & 906 & 8.9 \\
\hline $\begin{array}{l}\text { DA = densidad aparente, } \\
\text { orgánica. }\end{array}$
\end{tabular}

R. intraradices, 2) aislamiento San Rafael y 3) aislamiento Caracoles; con lo que se obtuvieron nueve tratamientos. Con los sustratos se llenaron las bolsas con capacidad de $5 \mathrm{~kg}$, perforándolas en la parte inferior para favorecer el drenaje, para luego colocarlas sobre bancales de fierro. Mientras que los hongos endomicorrízicos se aplicaron a razón de $4 \mathrm{~g}$ por maceta a $3 \mathrm{~cm}$ de profundidad para luego depositar la semilla.

Los tratamientos se distribuyeron mediante un diseño completamente al azar con cuatro repeticiones. Las variables evaluadas fueron: altura de planta, diámetro del tallo, número de hojas, biomasa seca de la raíz, tallo, hoja, peciolo y área foliar. Además de la colonización radical y el contenido de fósforo en el tejido vegetal a los 112 dds. La altura de planta se midió con una cinta métrica de la corona radical hasta la yema apical; el diámetro del tallo se midió a $5 \mathrm{~cm}$ de distancia de la corona radical hacia el ápice con un vernier digital (AutoTEC ${ }^{\mathrm{TM}}$ ) y el área foliar en $\mathrm{cm}^{2}$ con un integrador de área foliar (LI-COR, LI 3000 ${ }^{a}$ ). Mediante la técnica de tinción y clareo de Phillips y Hayman (1970) se prepararon 100 segmentos de raíz con longitud de 1.5 a 1.6 $\mathrm{cm}$ de cada muestreo, los cuales se observaron al microscopio óptico con el objetivo de 100X para identificar la colonización radical, los resultados se reportan en porcentaje de colonización micorrízica. La materia seca se determinó en una estufa de aire forzado por $72 \mathrm{~h}$ a temperatura de 75 a $80^{\circ} \mathrm{C}$. Los componentes del rendimiento de la parte aérea y radical se obtuvieron por pesado de la raíz, hojas, peciolo y tallo en una báscula (Ohaus, Adventurer Pro), mientras que el contenido de $\mathrm{P}$ se cuantificó en un espectrofotómetro Thermo Fisher Scientific Modelo $400 \frac{1}{4}$.

Con la biomasa seca se calcularon los siguientes parámetros fisiológicos: Tasa relativa de crecimiento $(\mathrm{TRC})=\left(\log _{e} \mathrm{~W}_{2}\right.$ (logaritmo del peso de biomasa seca 2) - $\log _{e} \mathrm{~W}_{1}$ (logaritmo del peso de biomasa seca 1)) / ( $T_{2}-T_{1}$ (Tiempo 2 - Tiempo 1)) de acuerdo con Milthorpe y Moorby (1982). Tasa de asimilación neta $(T A N)=\left(\mathrm{W}_{2}-\mathrm{W}_{1}\right.$ (Peso de biomasa seca 2 - Peso de biomasa seca 1)) $\left(\log _{e} \mathrm{AF}_{2}-\log _{e}\right.$ $\mathrm{AF}_{1}$ (Logaritmo del Área foliar en tiempo 2 - (logaritmo base 10 del peso de biomasa seca 2)) / $\left(\mathrm{AF}_{2}-\mathrm{AF}_{1}\right.$ (Área foliar en tiempo 2 - Área foliar en tiempo 1)) $\left(T_{2}\right.$ - $T_{1}$ (Tiempo 2 - Tiempo 1)) de acuerdo con Watson (1952). Relación raíz-vástago (R/V) se determinó con el peso seco de la parte aérea y el peso seco del sistema radical de acuerdo con Böhm (1979).

Los efectos entre tratamientos se determinaron por medio de análisis de varianza considerando los factores sustratos, microorganismos e interacciones de cada variable con el procedimiento PROC ANOVA y la comparación de medias de Tukey $(p \leq 0.05)$ con el programa computacional Statistical Analysis System, versión 8.1 (SAS 2002).

\section{RESULTADOS}

\section{Componentes morfológicos}

La altura de la planta tuvo diferencias estadísticas significativas entre tratamientos $(p \leq 0.05)$, las plantas más altas fueron las que crecieron en el sustrato con estiércol bovino y $R$. intraradices. Con diferencia de 6 y $21 \%$ entre Caracoles y San Rafael, respectivamente (Tabla 2). Entre sustratos, el que contiene estiércol bovino indujo mayor altura de planta $(50.3)$ y fue superior $(p \leq 0.05)$ a los otros dos sustratos. La altura promedio fue de $36.3 \mathrm{~cm}$ en suelo:arena, lo que representa $14 \mathrm{~cm}$ menos que las plantas que crecieron en el sustrato de suelo:estiércol. En el sustrato suelo:turba, las plantas registraron $4 \mathrm{~cm}$ menos altura que las plantas que 
Tabla 2. Comparación de medias en las variables morfológicas y fisiológicas de Tabebuia donnell-smithii Rose.

\begin{tabular}{|c|c|c|c|c|c|c|c|c|}
\hline & $\begin{array}{c}\text { Altura de } \\
\text { Planta }(\mathrm{cm})\end{array}$ & $\begin{array}{l}\text { Número } \\
\text { de Hojas }\end{array}$ & $\begin{array}{l}\text { Diámetro de } \\
\text { Tallo }(\mathrm{mm})\end{array}$ & Raíz & Tallo & $\begin{array}{l}\text { Lámina } \\
\text { foliar }\end{array}$ & Peciolo & $\begin{array}{c}\text { Área } \\
\text { foliar }\left(\mathrm{cm}^{2}\right)\end{array}$ \\
\hline Suelo:arena $+R$. intraradices & $37.75^{\star c}$ & $20.0^{a}$ & $7.58^{a b}$ & $3.87^{b c}$ & $3.75^{c d}$ & $4.70^{b}$ & $1.42^{b}$ & $1405^{b c}$ \\
\hline Suelo:arena Glomus sp San Rafael & $33.75^{c d}$ & $19.5^{a}$ & $7.18^{a b c}$ & $3.53^{c d}$ & $4.00^{c}$ & $4.69^{b}$ & $1.46^{b}$ & $1323^{b c}$ \\
\hline Suelo:arena Glomus sp Caracoles & $37.50^{c}$ & $19.5^{a}$ & $6.37^{b c d}$ & $2.85^{d e}$ & $3.30^{\text {cde }}$ & $4.27^{b}$ & $1.42^{b}$ & $1658^{b}$ \\
\hline Suelo:turba $+R$. intraradices & $29.75^{d}$ & $19.5^{a}$ & $6.05^{c d}$ & $2.33^{e f}$ & $2.68^{d e}$ & $4.04^{b}$ & $1.16^{b}$ & $1193^{c}$ \\
\hline Suelo:turba + Glomus sp San Rafael & $31.75^{c d}$ & $20.5^{a}$ & $5.71^{d}$ & $1.73^{f}$ & $2.49^{e}$ & $3.84^{b}$ & $1.18^{b}$ & $1259^{c}$ \\
\hline Suelo:turba + Glomus sp Caracoles & $37.00^{c}$ & $21.5^{a}$ & $6.02^{c d}$ & $1.70^{f}$ & $2.41^{e}$ & $3.73^{b}$ & $1.16^{b}$ & $1214^{c}$ \\
\hline Suelo:estiércol bovino $+R$. intraradices & $54.75^{a}$ & $22.0^{a}$ & $8.05^{a}$ & $4.30^{b}$ & $8.93^{a}$ & $13.09^{a}$ & $4.39^{a}$ & $3399^{a}$ \\
\hline Suelo:estiércol bovino + Glomus sp San Rafael & $45.12^{b}$ & $22.0^{a}$ & $7.94^{a}$ & $5.13^{a}$ & $7.52^{b}$ & $12.51^{a}$ & $3.75^{a}$ & $3073^{a}$ \\
\hline Suelo:estiércol bovino + Glomus sp Caracoles & $51.25^{a b}$ & $22.0^{a}$ & $7.88^{a}$ & $4.44^{a b}$ & $7.18^{b}$ & $11.82^{a}$ & $4.32^{a}$ & $3288^{a}$ \\
\hline CV (\%) & 7.51 & 8.66 & 8.76 & 8.97 & 10.01 & 8.99 & 11.97 & 8.37 \\
\hline
\end{tabular}

crecieron en suelo:arena y $17.7 \mathrm{~cm}$ menos que las que crecieron en sustrato suelo:estiércol. Los hongos endomicorrízicos $R$. intraradices y Glomus sp Caracoles fueron estadísticamente diferentes $(p \leq 0.05)$ a Glomus sp San Rafael. El número de hojas presentó diferencias estadísticas $(p \leq 0.05)$ solamente entre sustratos, siendo los sustratos suelo:estiércol y suelo:turba superiores al sustrato suelo:arena, con incremento de tres y una hoja, respectivamente.

El diámetro del tallo mostró diferencias estadísticas $(p \leq 0.05)$ durante el periodo de evaluación. Entre sustratos, el estiércol bovino indujo mayor grosor de tallo ( $p \leq 0.05$ ), pero no se detectaron diferencias estadísticas entre los hongos endomicorrízicos. La interacción de estiércol bovino biofertilizado con $R$. intraradices indujo el mayor incremento de grosor de tallo.

\section{Componentes Fisiológicos}

El peso seco radical presenta la variación más contrastante en respuesta a los microorganismos y sustratos (Tabla 2). Entre sustratos, el suelo:estiércol bovino incrementó la biomasa radial 35 y $140 \%$ más en comparación con suelo:arena y suelo:turba. Los microorganismos $R$. intraradices y Glomus sp San Rafael presentaron $16 \%$ más peso seco de raíz, que Glomus sp caracoles $(p \leq 0.05)$. El peso seco del tallo incrementó con el sustrato con estiércol bovino y se mejoró con la aplicación de $R$. intraradices ( $\mathrm{p} \leq$ 0.05). Entre sustratos el estiércol bovino incrementó 311 y $214 \%$ la biomasa del tallo en comparación con suelo:turba y suelo:arena, respectivamente. El hongo $R$. intraradices fue más consistente con incremento del $9 \%$ del grosor del tallo en la planta hospedera en comparación con los dos aislamientos.

El peso seco de la lámina foliar aumentó en el sustrato con estiércol bovino y $R$. intraradices, mismo que fue estadísticamente superior a los otros tratamientos $(p \leq 0.05)$. La biomasa promedio fue de $12.4 \mathrm{~g}$ en el sustrato con estiércol, $3.8 \mathrm{~g}$ en suelo:turba y $4.5 \mathrm{~g}$ en suelo:arena. El peso seco del peciolo de las hojas tuvo la misma tendencia que la biomasa en la lámina foliar, entre sustratos y microorganismos. El mayor incremento se tuvo en el sustrato con estiércol bovino y $R$. intraradices ( $\leq 0.05$ ).

El área foliar de la planta tuvo comportamiento semejante a la materia seca de la lámina foliar y el peso seco del peciolo (Tabla 3). Lo que indica desarrollo concomitante de estos componentes del rendimiento. Entre los sustratos, el mayor incremento del área foliar se presentó con la combinación de estiércol bovino en interacción con los tres microorganismos, y fue estadísticamente diferente $(p$ $\leq 0.05)$ a la interacción de los otros dos sustratos, con los mismos microorganismos. El suelo:estiércol incrementó el área foliar en 122 y 166\% en comparación con los sustratos suelo:arena y suelo:turba, respectivamente.

La Tasa relativa de crecimiento (TRC) de 28 a 56 dds presenta valores promedio altos de 0.104 g.día ${ }^{-1}$ en todos los tratamientos, y los mismos tienden a disminuir con la edad de la planta de 56 a 84, a 0.059 y de 84 a 112 días a $0.040 \mathrm{~g} \cdot$ día $^{-1}$, independientemente del sustrato y microorganismo. Al inicio se incrementa la producción de biomasa de forma eficiente y sostenida en respuesta al fortalecimiento del 
Tabla 3. Comparaciones de factores sustrato y hongo endomicorrízico en Tabebuia donnell-smithii Rose.

\begin{tabular}{ccccccccc}
\hline Sustrato & $\begin{array}{c}\text { Altura de } \\
\text { Planta }(\mathrm{cm})\end{array}$ & $\begin{array}{c}\text { Número } \\
\text { de Hojas }\end{array}$ & $\begin{array}{c}\text { Diámetro de } \\
\text { Tallo }(\mathrm{mm})\end{array}$ & Raíz & Tallo & $\begin{array}{c}\text { Lámina } \\
\text { foliar }\end{array}$ & $\begin{array}{c}\text { Peciolo } \\
(\mathrm{g})\end{array}$ & $\begin{array}{c}\text { Área } \\
\text { foliar }\left(\mathrm{cm}^{2}\right)\end{array}$ \\
\hline Suelo:arena & $36.3^{\star b}$ & $19.6^{b}$ & $7.04^{b}$ & $3.42^{b}$ & $3.68^{b}$ & $4.56^{b}$ & $1.43^{b}$ & $1462^{b}$ \\
Suelo:turba & $32.8^{c}$ & $20.5^{a b}$ & $5.99^{c}$ & $1.92^{c}$ & $2.53^{c}$ & $3.87^{c}$ & $1.17^{b}$ & $1222^{c}$ \\
Suelo:estiércol bovino & $50.3^{a}$ & $22.0^{a}$ & $7.96^{a}$ & $4.62^{a}$ & $7.88^{a}$ & $12.4^{a}$ & $4.15^{a}$ & $3253^{a}$ \\
Hongo R. intraradices & $40.7^{a}$ & $20.5^{a}$ & $7.23^{a}$ & $3.50^{a}$ & $5.12^{a}$ & $7.28^{a}$ & $2.32^{a}$ & $1999^{a b}$ \\
Glomus sp San Rafael & $36.8^{b}$ & $20.6^{a}$ & $6.94^{a}$ & $3.46^{a}$ & $4.67^{b}$ & $6.78^{a}$ & $2.13^{a}$ & $1885^{b}$ \\
Glomus sp Caracoles & $41.9^{a}$ & $21.0^{a}$ & $6.75^{a}$ & $3.00^{b}$ & $4.30^{b}$ & $6.84^{a}$ & $2.30^{a}$ & $2035^{a}$ \\
\hline
\end{tabular}

aparato fotosintético (Figura 1).

La tasa de asimilación neta (TAN) fue mayor de 28 a 56 días en todos los tratamientos. Entre sustratos, en este mismo periodo, el suelo más estiércol bovino presenta la TAN más baja de 0.24 a 0.28 en comparación con los otros sustratos, que fue de 0.36 a $0.37 \mathrm{~g} \cdot \mathrm{cm}^{2} \cdot$ día $\cdot{ }^{1}$, pero en los periodos de 56 a 84 y 84 a 112 días se incrementa la TAN en el sustrato con estiércol de bovino en combinación con los microorganismos (Figura 2). Los microorganismos $R$. intraradices y Glomus sp San Rafael presentaron incremento de la TAN en la evaluación de 56 a 84 dds y disminuyó de 84 a 112 dds. La especie de Glomus sp caracoles presenta la TAN más baja de 84 a 112 días, pero es mayor de 28 a 56 días.

\section{Contenido de fósforo}

El contenido de fósforo incrementó en el tejido vegetal de Tabebuia donnell-smithii Rose con los tres hongos endomicorrízicos en interacción con la adición al suelo de estiércol bovino (Figura 3). Mientras que con $R$. intraradices se presenta la concentración más alta de fósforo en los tres sustratos.

\section{DISCUSIÓN}

\section{Sustratos}

Los sustratos presentaron variaciones importantes en contenido de N, P, K y MO. Se incrementaron con la adición de turba y estiércol, pero en éste último, los valores de $\mathrm{P}$ y $\mathrm{K}$ representan entre 120 y $365 \%$ con relación con el sustrato adicionado con turba. Estas concentraciones indujeron mayor crecicimiento vegetal en la planta huésped con efecto diferencial en la capacidad de transporte por los hon- gos endomicorrízicos evaluados. Los hongos endomicorrízicos favorecen el transporte de $\mathrm{N}$ (Leigh et al. 2009), $\mathrm{P}$ y otros nutrimentos (Smith et al. 2015), y con ello cambios en la actividad fotosintética y crecimiento de las plantas (Barea et al. 2002).

\section{Componentes morfológicos}

El incremento en altura de planta probablemente se favoreció con el aumento del $\mathrm{N}$, $\mathrm{P}$ y $\mathrm{K}$, en el sustrato suelo:estiércol y $R$. intraradices. La especie $R$. intraradices promueve mayor altura en plantas perennes como en Cedrela odorata L. con crecimientos de hasta $20.7 \mathrm{~cm}$ más al adicionar $30 \%$ de estiércol bovino al suelo, en comparación con el testigo (Aguirre-Medina et al. 2014b). En general, los hongos endomicorrízicos tiene la capacidad de explorar mayor volumen de suelo y de esta manera, abastecer de nutrientes a la planta (Leigh et al. 2009), como fósforo (Garza-Cano et al. 2005) y nitrógeno (Ibarra-Puón et al. 2014). La poca variación entre tratamientos en el número de hojas ha sido reportada en sustratos con Tabebuia donnell-smithii Rose (Aguirre-Medina et al. 2014a). Pero en pocas plantas como Theobroma cacao L. se ha incrementado el número de hojas con $R$. intraradices (Aguirre-Medina et al. 2007). En algunas asociaciones simbióticas la demanda de carbohidratos puede ser alta por parte del microorganismo en la etapa inicial de establecimiento. En esta etapa disminuye la disponibilidad de carbohidratos hacia el vástago, debido a que los compuestos son requeridos por la raíz (Roveda y Polo 2007).

Las diferencias en el grosor del tallo se pueden deber a la influencia de los sustratos, especialmente con suelo:estiércol con aporte de 

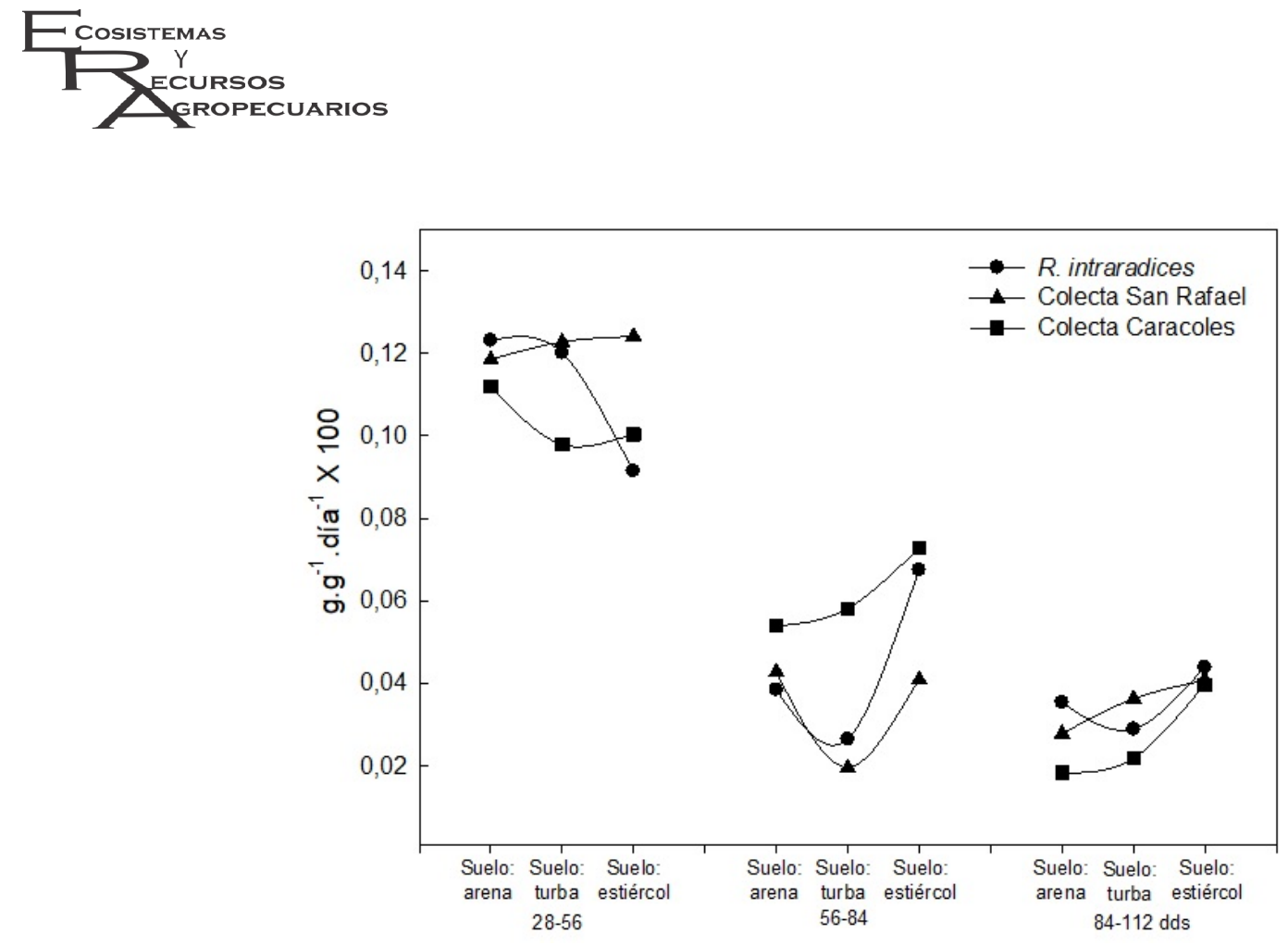

Figura 1. Dinámica de la tasa media relativa de crecimiento (TRC) en Tabebuia donnell-smithii Rose.

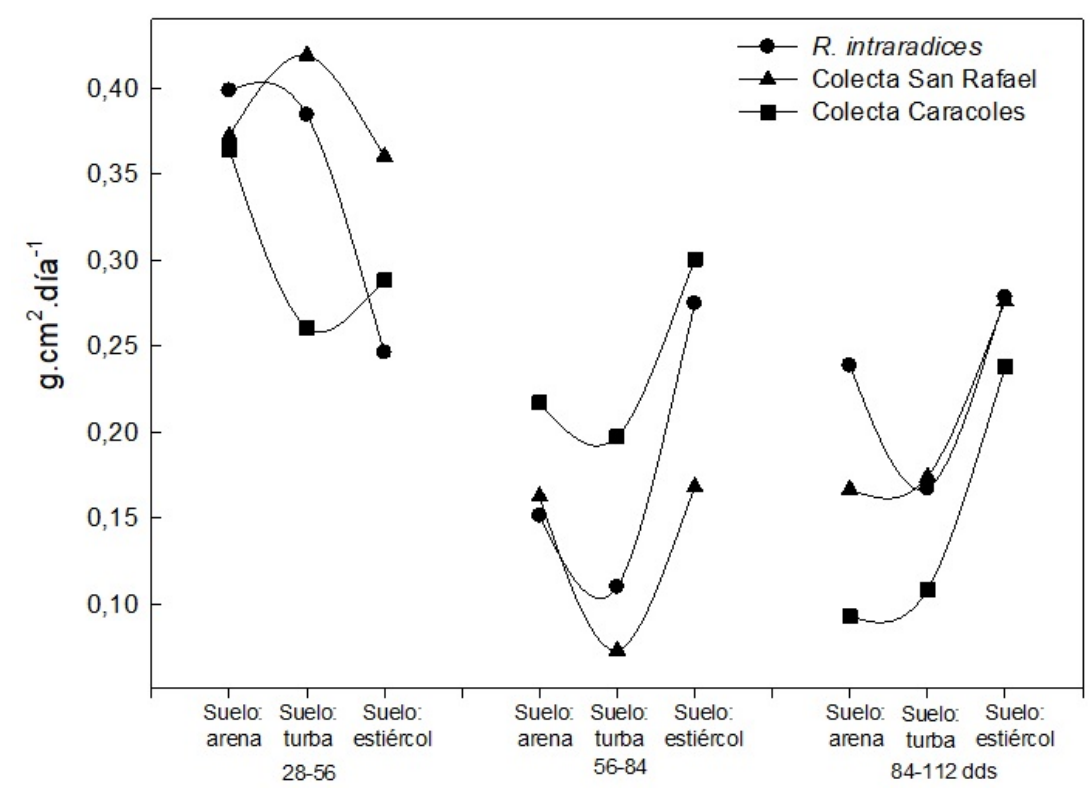

Figura 2. Dinámica de la tasa media de asimilación neta (TAN) en Tabebuia donnell-smithii Rose.

microorganismos. Aun cuando se ha indicado que la simbiosis micorrízica arbuscular carece de especificidad taxonómica (Cuenca et al. 2007), parece que se presenta cierta compatibilidad funcional entre la planta, sustrato y hongo. Esta in- teracción genera preferencia en la combinación de microorganismos-planta hospedera que se expresa en mayor crecimiento vegetal. Para Tectona grandis L. F. y Astronium graveolens Jacq. el incremento en diámetro del tallo se tuvo con Glomus fascicu- 


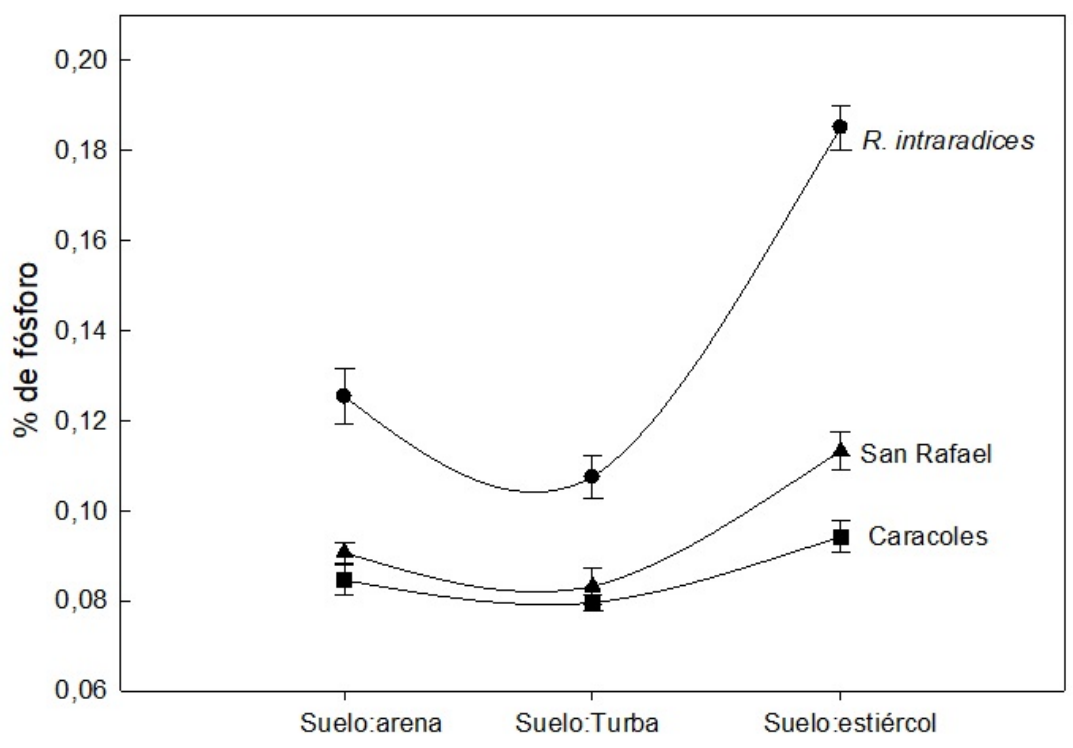

Figura 3. Contenido de fósforo $(\mathrm{P})$ en Tabebuia donnell-smithii Rose biofertilizada con diversos sustratos y hongos endomicorrízicos en condiciones de vivero. La línea vertical indica \pm el error estándar de cuatro repeticiones.

latum (Thaxter) Gerdemann \& Trappe con 11.8\% más que el testigo (Hernández y Salas 2009). En Coffea canephora (P.) ex Froehner, el diámetro del tallo incrementó cuando se inoculó plantas con $R$. intraradices en el sustrato suelo:arena (Ibarra-Puón et al. 2014). En otros cultivos perennes se reporta este mismo efecto con $G$. intraradices (Aguirre-Medina et al. 2011). La colonización radical de los aislamientos fue de $53 \%$ con San Rafael y $49 \%$ con Caracoles, y promovieron en forma diferencial el crecimiento de los diversos componentes morfológicos del rendimiento en $T$. donnell-smithii Rose.

\section{Componentes fisiológicos}

La disminución de la biomasa radical al biofertilizar con $R$. intraradices y usar como sustrato suelo más estiércol bovino, probablemente se deba a la sustitución del crecimiento de los pelos radicales por el crecimiento de la hifa del hongo. Al parecer la hifa del hongo sustituye los pelos de la raíz y la planta transporta más fotosintatos a la parte aérea para la producción de biomasa, mientras que los hongos endomicorrízicos al extender el crecimiento externo del micelio actúan como extensión de la superficie de absorción de la raíz (Leigh et al. 2009). A los 84 y 112 días, la acumulación de materia seca total y la colonización radical aumentaron con $R$. intraradices. Resultados similares fueron reportados por Ibarra-Puón et al. (2014) para C. canephora (P.) ex Froehner con el mismo hongo endomicorrízico. Mientras que en Phaseolus vulgaris L. var. Michoacán 12-A-3 se reporta menor crecimiento radical con la mezcla de Glomus intraradices y G. macrocarpum Tulase et Smith (Aguirre-Medina y Kohashi-Shibata 2002).

En otros cultivos perennes biofertilizados con $R$. intraradices en vivero se reportan modificaciones en la asignación de biomasa radical de la planta hospedera en cantidad y tiempo. Para $C$. arabica $L$ se reporta incremento del sistema radical (Aguirre-Medina et al. 2011), y para Theobroma cacao $L$ se reporta disminución (Aguirre-Medina et al. 2007). Mientras que para T. donnell-smithii Rose biofertilizada con aislamientos endomicorrízicos del sur de Chiapas se incrementa la biomasa radical a los 56 y 84 dds (Aguirre-Medina et al. 2014a). En tanto que en Coffea canephora (P.) ex Froehner se incrementó el crecimiento del sistema radical de las plantas que crecieron en el sustrato suelo:arena, con mayor colonización micorrízica en el tratamiento con menor crecimiento radical (Ibarra-Puón et al. 2014). El incre- 
mento en el crecimiento de la raíz puede estar relacionado con el incremento de algunas sustancias del crecimiento, producto de la simbiosis, como sucedió con las giberelinas en la raíz de Bouteloa gracilis (Willd. ex Kunth) Lag. ex Griffiths colonizando con Glomus fasciculatum (Thaxter) Gerdemann \& Trappe (Allen et al. 1982).

El incremento de la biomasa del tallo en el sustrato con suelo más estiércol bovino, es probable que se deba a la capacidad de producción de enzimas hidrolíticas, como proteasas y fosfatasas de la hifa extra matricial, las cuales pueden tener un impacto importante en la mineralización de la MO y la disponibilidad de nutrientes (Sylvia 2005). Para Coffea canephora (P.) ex Froehner la mayor acumulación de biomasa en el tallo se reporta con el sustrato suelo:arena con $9.16 \%$ de MO (Ibarra-Puón et al. 2014). La simbiosis endomicorrízica genera un sistema radical complementario que favorece el aporte de nutrientes y agua a la planta y, con ello cambios en su fisiología de la planta hospedera (Barea et al. 2002). La inducción de mayor biomasa en lámina foliar con el sustrato suelo más estiércol bovino se favoreció por su alto contenido nutrimental. En Cedrela odorata L, se reporta incremento de biomasa de la lámina foliar en suelo adicionado con $30 \%$ de estiércol bovino y con el hongo $R$. intraradices (AguirreMedina et al. 2014). La inducción de mayor desarrollo de la lámina foliar con $R$. intraradices ha sido reportada en cultivos anuales (Aguirre-Medina 2006).

El peso seco del peciolo también se incrementa en las plantas que crecieron en el sustrato con suelo:estiércol bovino, el cual contiene mayor contenido de fósforo y potasio. Al respecto, AguirreMedina et al. (2007) reportan incremento del peso del peciolo de las hojas de T. cacao L. con Azospirillum brasilense y menor crecimiento con $R$. intraradices. La mayor área foliar en las plantas que crecieron en el sustrato con estiércol bovino, sugiere interacción entre los hongos endomicorrízicos y los sustratos ricos en fósforo y potasio. Al respecto Sylvia (2005) reporta mayor biomasa foliar en la planta hospederacon hongos micorrízicos, por incremento de la actividad fotosintética. En el caso de $R$. intraradices, también se incrementó la biomasa foliar en condiciones de vivero en T. cacao L. y Coffea arabica L. (Aguirre-Medina et al. 2007, 2011). La alta tasa relativa de crecimiento (TRC) inicial y la disminución en las siguientes etapas, se debe al aumento gradual de tejido no asimilatorio, como sucede en otras plantas anuales (AguilarGarcía et al. 2005). El incremento inicial en el número de hojas con los tratamientos biofertilizados, puede deberse a la respuesta diferencial de la planta con el microorganismo en la demanda de mayores fotosintatos en el sistema radical y la menor asignación para formar biomasa en la parte aérea. En la variación de la TRC están involucrados factores internos, como la etapa de desarrollo de la planta en interacción con factores externos bióticos y abióticos (Shipley 2006). Algunos reportes indican que el tipo de fertilización puede tener impacto en el crecimiento de las plantas (Derkowska et al. 2015), como el fósforo en maíz, que incrementa la tasa de crecimiento (Díaz-López et al. 2013). El crecimiento es mayor con sustratos ricos en nutrientes, especialmente con fósforo, que es el más transportado por los hongos endomicorrízicos (Andrade et al. 2009, Tajin y Drevon 2012). Es probable, que la respuesta en la tasa de crecimiento esté relacionada con la capacidad del hongo endomicorrízico en transportar fósforo a la planta. La respuesta de la TAN sugiere inducción diferencial de los microorganismos en el incremento de la biomasa de T. donnell-smithii Rose, como respuesta al crecimiento modular y la distribución de los recursos, o bien, en las diferencias en la forma de explotarlos (Collado-Vides 1997). La TAN depende del área foliar, la disposición y la edad de las hojas, así como de su metabolismo interno y la respuesta a factores externos mediante la actividad respiratoria (Hunt et al. 2002). Al incrementarse el área foliar, se mejora la TAN (Aguilar et al. 2006), el incremento del área foliar con los tres microorganismos sugiere mejora en el transporte de nutrientes, especialmente con fósforo. Al respecto Aguirre-Medina et al. (2007) reportan el incremento de la TAN al aplicar G. intraradices en Theobroma cacao L.

\section{Contenido de fósforo}

Uno de los principales beneficios que las plantas reciben en la simbiosis con los hongos 
micorrízicos es la aportación de fósforo, nutriente que es más transportado por los hongos micorrízicos. Al respecto, se sabe que las hifas fúngicas tienen mayor afinidad con el ión fosfato cuando su concentración es baja en la solución (Andrade et al. 2009, Tajini y Drevon 2012). Por lo que en sistemas de producción de bajos insumos la actividad de la micorriza es más efectiva (Grant et al. 2005), como en suelos de baja fertilidad (Mudge et al. 2003). Para T. donnell-smithii Rose los hongos endomicorrízicos pueden facilitar el transporte del fósforo a la planta, por lo que se sugiere reducir la utilización de fertilizantes fosfatados en los viveros cuando se aplican hongos endomicorrízicos. En las plantas de C. arabica se tuvieron resultados similares al incrementar la absorción de fósforo con G. intraradices (Aguirre-Medina et al.
2011), Araucaria angustifolia (Bertol.) Kuntze y con $R$. intraradices (Moreira-Souza y Cardoso 2002).

\section{CONCLUSIONES}

Los hongos endomicorrízicos promovieron el crecimiento vegetal de $T$. donnell-smithii Rose con aumento en la asignación de materia seca en los componentes fisiológicos y morfológicos del rendimiento. El incremento fue mayor en suelo con $20 \%$ de estiércol bovino. La biofertilización con $R$. intraradices promueve el crecimiento vegetal de tallo y área foliar. El contenido de fósforo en el tejido vegetal incrementó con los hongos endomicorrízicos, pero fue mayor cuando se biofertilizó con $R$. intraradices.

\section{LITERATURA CITADA}

Aguilar-García L, Escalante-Estrada A, Fucikovsky-Zak L, Tijerina-Chávez L, Engleman EM (2005) Área foliar, tasa de asimilación neta, rendimiento y densidad de población en girasol. Terra Latinoamericana 23: 303310.

Aguilar LMG, Carrillo SAJ, Rivera PA, González HVA (2006) Análisis de crecimiento y relaciones fuente demanda en dos variedades de papa (Solanum tuberosum L.). Revista Fitotecnia Mexicana 29: 145-156.

Aguirre-Medina JF, Kohashi-Shibata J (2002) Dinámica de la colonización micorrízica y su efecto sobre los componentes del rendimiento y el contenido de fósforo en frijol común. Agricultura Técnica en México 28: 23-33.

Aguirre-Medina JF (2006) Biofertilizantes microbianos: Experiencias agronómicas del programa nacional del INIFAP en México. Primera Edición. Instituto Nacional de Investigaciones Forestales, Agrícolas y Pecuarias. México. 201p.

Aguirre-Medina JF, Mendoza-López A, Cadena-Iñiguez J, Avendaño-Arrazate CH (2007) La biofertilización del cacao (Theobroma cacao L.) en vivero con Azospirillum brasilense Tarrand, Krieg et Döbereiner y Glomus intraradices Schenk et Smith. Interciencia 32: 1-6.

Aguirre-Medina JF, Moroyoqui-Ovilla DM, Mendoza-López A, Cadena-Iñiguez J, Avendaño-Arrazate CH, AguirreCadena JF (2011) Aplicación de A. brasilense y G. intraradices a Coffea arabica en vivero. Agronomía Mesoamericana 22: 1-10.

Aguirre-Medina JF, Aguirre-Cadena JF, Cadena-Iñiguez J, Avendaño-Arrazate CH (2012) Biofertilización en plantas de la selva húmeda tropical. 1a edición. Colegio de Postgraduados. México. 99p.

Aguirre-Medina JF, Culebro-Cifuentes F, Cadena-Iñiguez J, Aguirre-Cadena JF (2014a) Crecimiento de Tabebuia Donnell-Smithii Rose inoculada con hongos micorrízicos y Azospirillum brasilense. Agrociencia 48: 331345.

Aguirre-Medina JF, Mina-Briones FO, Cadena-Iñiguez J, Dardón-Zunun JD, Hernández-Sedas DA (2014b) Crecimiento de Cedrela odorata L. Biofertilizada con Rhizophagus intraradices y Azospirillum brasilense en vivero. Revista Chapingo Serie Ciencias Forestales y del Ambiente XX: 177-186. 
Allen MF, Moore Jr TS, Christensen M (1982) Phytohomone change in Bouteloua gracilis infected by vesiculararbuscular mycorrhizal. II. Altered levels of gibberellin-like substances and abscisic acid in the host plant. Canadian Journal of Botany 60: 468-471.

Andrade SAL, Mazzafera P, Schiavinato MA, Silveira APD (2009) Arbuscular mycorrhizal association in coffee. Journal of Agricultural Science 147: 105-115.

Barea JM, Azcon R, Azcon-Aguilar C (2002) Mycorrhizosphere interactions to improve plant fitness and soil quality. Antonie Van Leeuwenhoek International Journal of General and Molecular Microbiology 81: 343351.

Böhm W (1979) Methods of studying root systems. Spring-verlag. Germany. 188p.

Collado-Vides $L$ (1997) Aspectos ecológicos y evolutivos de la arquitectura modular en plantas: perspectivas en algas marinas. Revista Chilena de Historia Natural 70: 23-39.

Cuenca G, Cáceres A, Oirdobro G, Hasmy Z, Urdaneta C (2007) Las micorrizas arbusculares como alternativa para una agricultura sustentable en áreas tropicales. Interciencia 32: 23-29.

Díaz-López E, Loeza-Corte JM, Campos-Pastelín JM, Morales-Rosales EJ, Domínguez-López A, Franco-Mora O (2013) Eficiencia en el uso de la radiación, tasa de asimilación neta e integral térmica en función del fósforo en maíz (Zea mays L.). Agrociencia 47: 135-146.

Doubková P, Vlasáková E, Sudová R (2013) Arbuscular mycorrhizal symbiosis alleviates drought stress imposed on Knautia arvensis plants in serpentine soil. Plant and Soil 370: 149-161.

Derkowska E, Sas-Paszt L, Trzciński P, Przybył M, Weszczak K (2015) Influence of biofertilizers on plant growth and rhizosphere microbiology of greenhouse-grown strawberry cultivars. Acta Scientiarum Polonorum Hortorum Cultus 14: 83-96.

Garza-Cano I, Pecina-Quintero V, Díaz-Franco A, Williams-Alanís H, Ramírez-De León JA (2005) Sorgo cultivado con biofertilizantes, fitohormonas y fósforo inorgánico. Terra Latinoamericana 23: 581-586.

Gianinazzi S, Gollotte A, Binet MN, van Tuinen D, Redecker D, Wipf D (2010) Agroecology: the key role of arbuscular mycorrhizas in ecosystem services. Mycorrhiza 20: 519-530.

Gerdemann JW, Nicholson TH (1963) Spores of mycorrhizal Endogone species extracted from the soil by wet sieving and decanting. Transactions of the British Mycological Society 46: 235-244.

Grant C, Bittman S, Montreal M, Plenchette C, Morel C (2005). Soil and fertilizer phosphorus: Effects on plant P supply and mycorrhizal development. Canadian Journal of Plant Science 85: 3-14.

Hernández W, Salas E (2009) La Inoculación con Glomus fasciculatum en el crecimiento de cuatro especies forestales en vivero y campo. Agronomía Costarricense 33: 17-30.

Hunt R, Causton DR, Shipley B, Askew AP (2002) A modern tool for classical plant growth analysis. Annals of Botany 90: 485-488.

Ibarra-Puón JC, Aguirre-Medina JF, Ley-De Coss A, Cadena-Iñiguez J, Zavala-Mata A (2014) Inoculación de Coffea canephora (Pierre) ex Froehner con Rhizophagus intraradices (Schenck et Sm.) Walker et Schuessler y Azospirillum brasilense Tarrand, Krieg et Döbereiner en vivero. Revista Chapingo Serie Horticultura 20: 201-213.

INEGI (2005) Marco Geoestadístico Municipal, versión 3.1. Prontuario de información geográfica municipal de los Estados Unidos Mexicanos. México. http://www3.inegi.org.mx/sistemas/mexicocifras/datos-geograficos/21/ 21158.pdf. Fecha de consulta: 1 de marzo del 2017. 
Jaizme-Vega MC, Rodríguez-Romero AS (2008) Integración de microorganismos benéficos (Hongos micorrícicos y bacterias rizosféricas) en agrosistemas de las Islas Canarias. Agroecología 3: 33-39.

Leigh J, Hodge A, Fitter AH (2009) Arbuscular mycorrhizal fungi can transfer substantial amounts of nitrogen to their host plant from organic material. New Phytologist 181: 199-207.

Milthorpe FL, Moorby J (1982) Introducción a la fisiología de los cultivos. Ed. Hemisferio Sur. Argentina. 259p

Moreira-Souza M, Cardoso EJBN (2002) Dependência Micorrízica de Araucaria angustifolia (Bert.) O. Ktze. sob doses de fósforo. Revista Brasileira de Ciência do Solo 26: 905-912.

Mudge SR, Smith FW, Richardson AE (2003) Root-specific and phosphate-regulated expression of phytase under the control of a phosphate transporter promoter enables Arabidopsis to grow on phytate as a sole P source. Plant Science 165: 871-878.

Phillips JM, Hayman DJ (1970) Improved procedures for clearing and staining parasitic and vesicular-arbuscular mycorrhizal fungi for rapid assessment of infection. Transactions of the British Mycological Society 55: 158-161.

Roveda R, Polo C (2007) Mecanismos de adaptación de maíz asociado a Rhizophagus spp. en suelos con bajo fósforo disponible. Agronomía Colombiana 25: 349-356.

Read D (1998) Plants on the web. Nature 396: 22-23.

SAS (2002) System for Windows version 9.0. Statistical Analysis System. SAS Institute Inc. Cary NC 27513, USA. 5136p.

Serralde OAM, Ramírez GMM (2004) Análisis de poblaciones de micorrizas en maíz (Zea mays) cultivado en suelos ácidos bajo diferentes tratamientos agronómicos. Revista Corpoica 5: 31-40.

Shipley B (2006) Net assimilation rate, specific leaf area and leaf mass ratio: which is most closely correlated with relative growth rate? A meta-analysis. Functional Ecology 20: 565-574.

Smith S, Anderson I, Smith F (2015) Mycorrhizal associations and phosphorus acquisition: from cells to ecosystems. Annual Plants Review 48: 409-440.

Sylvia MD (2005) Mycorrhizal symbioses. In: Sylvia MD, Fuhrmann JJ, Harte GP, Zuberer AD (eds). Principles and applications of soil microbiology. Second Edition. Pearson Prentice Hall. New Jersey, USA. pp. 263282.

Tajini F, Drevon JJ (2012) Phosphorus use efficiency in common bean (Phaseolus vulgaris L.) as related to compatibility of association among arbuscular mycorrhizal fungi and rhizobia. African Journal of Biotechnology 11: $12173-12182$.

Wang B, Qiu Y-L (2006) Phylogenetic distribution and evolution of mycorrhizas in land plants. Mycorrhiza 16: 299-363.

Watson DJ (1952) The physiological basis of variation in yield. Advances in agronomy 4: 101-145. 
